\title{
BMJ Open Antidepressants and the risk of hyponatremia: a Danish register-based population study
}

\author{
Katja Biering Leth-Møller, ${ }^{1}$ Annette Højmann Hansen, ${ }^{2}$ Maia Torstensson, ${ }^{1}$ \\ Stig Ejdrup Andersen, ${ }^{3}$ Lars Ødum, ${ }^{4}$ Gunnar Gislasson, ${ }^{5}$ Christian Torp-Pedersen, ${ }^{6}$ \\ Ellen Astrid Holm ${ }^{1}$
}

To cite: Leth-Møller KB, Hansen AH, Torstensson M, et al. Antidepressants and the risk of hyponatremia: a Danish register-based population study. BMJ Open 2016;6: 011200.

doi:10.1136/bmjopen-2016011200

- Prepublication history and additional material is available. To view please visit the journal (http://dx.doi.org/ 10.1136/bmjopen-2016011200).

Received 25 January 2016 Revised 29 March 2016 Accepted 28 April 2016 CrossMark

${ }^{1}$ Department of Geriatric Medicine, Nykøbing Falster Hospital, Nykøbing Falster, Denmark

${ }^{2}$ Department of Geriatric Medicine, Slagelse Hospital, Slagelse, Denmark

${ }^{3}$ Clinical Pharmacology Unit, University Hospital Zealand, Roskilde, Denmark

${ }^{4}$ Department of Biochemistry, University Hospital Zealand, Roskilde, Denmark

${ }^{5}$ Department of Cardiology, Gentofte Hospital, Hellerup, Denmark

${ }^{6}$ Institute of Health, Science and Technology, Aalborg University, Aalborg, Denmark

Correspondence to Dr Katja Leth-Møller; katja. biering.leth-moeller.01@ regionh.dk

\section{ABSTRACT}

Objective: To examine the association between classes of antidepressants and hyponatremia, and between specific antidepressants and hyponatremia. Design: Retrospective register-based cohort study using nationwide registers from 1998 to 2012.

Setting: The North Denmark Region.

Participants: In total, 638352 individuals were included.

\section{Primary and secondary outcome measures:}

Plasma sodium was obtained from the LABKA database. The primary outcome was hyponatremia defined as plasma sodium ( $p$-sodium) below $135 \mathrm{mmol} / \mathrm{L}$ and secondary outcome was severe hyponatremia defined as p-sodium below $130 \mathrm{mmol} / \mathrm{L}$. The association between use of specific antidepressants and hyponatremia was analysed using multivariable Poisson regression models.

Results: An event of hyponatremia occurred in 72509 individuals and $11.36 \%(n=6476)$ of these events happened during treatment with antidepressants. Incidence rate ratios and $\mathrm{Cls}$ for the association with hyponatremia in the first $\mathrm{p}$-sodium measured after initiation of treatment were for citalopram 7.8 (Cl 7.42 to 8.20); clomipramine 4.93 (Cl 2.72 to 8.94); duloxetine 2.05 ( $\mathrm{Cl} 1.44$ to 292 ); venlafaxine 2.90 (Cl 2.43 to 3.46 ); mirtazapine 2.95 ( $\mathrm{Cl} 2.71$ to 3.21 ); and mianserin 0.90 (CI 0.71 to 1.14).

Conclusions: All antidepressants except mianserin are associated with hyponatremia. The association is strongest with citalopram and lowest with duloxetine, venlafaxine and mirtazapine.

\section{BACKGROUND}

The consumption of antidepressants has increased significantly in most Organisation for Economic Co-operation and Development (OECD) countries since 2000. Denmark is among the OECD countries with the highest consumption. ${ }^{1}$

Hyponatremia is one of the many wellknown side effects of antidepressants. ${ }^{2}{ }^{3}$ Even mild hyponatremia is associated with instability and falls, reduced cognitive function, osteoporosis and increased morbidity and mortality. ${ }^{4-13}$

\section{Strengths and limitations of this study}

- Large population study including all individuals living in The North Denmark Region.

- Owing to the large number of individuals included, it was possible to compare strengths of associations between specific antidepressants and occurrence of hyponatremia.

- Linking between several national registers of high quality made it possible to adjust for comorbidity and limited missing data.

- Observational design.

- Confounding by indication cannot be fully excluded.

The kidneys control the renal excretion of water and electrolytes and keep serum osmolality and sodium within a narrow range. Several hormones influence the renal handling of water and electrolytes, the most important being arginine vasopressin, also known as antidiuretic hormone (ADH).

$\mathrm{ADH}$ binds to $\mathrm{V} 2$ receptors in the kidney, and thereby leads to an increase in reabsorption of water in the collecting ducts. The main stimuli for $\mathrm{ADH}$ is plasma osmolality and low plasma volume, but a number of nonspecific stimuli like stress and nausea can also result in elevated ADH concentrations. ${ }^{14} 15$

The presence of hypotonic hyponatremia always implies excess of water, and hyponatremia essentially is a water balance disorder. ${ }^{16} \mathrm{In}$ most cases of hypotonic hyponatremia, ADH secretion is increased due to one of the following reasons: (1) appropriate response to decreased effective plasma volume as in hypovolaemia, cirrhosis and heart failure; (2) ectopic production as seen in neoplastic diseases resulting in syndrome of inappropriate ADH (SIADH); or (3) syndrome of inappropriate antidiuresis (SIAD) either due to a drug induced increase in $\mathrm{ADH}$ production (SIADH) or an enhanced renal response to $\mathrm{ADH}$, the nephrogenic syndrome of antidiuresis. ${ }^{16}$ 
In a cross-sectional study of elderly users of antidepressants, Mannesse et al studied the mechanisms of hyponatremia in 34 patients with hyponatremia. Data on serum and urinary sodium were available in 30 patients, and of these 23 were classified as having either SIADH or probable SIADH. However, only in a minority of these patients was ADH not suppressed, and the authors concluded that the mechanism of hyponatremia produced by antidepressants might be either an elevated ADH or an increased renal response to $\mathrm{ADH} .{ }^{17}$ Some previous studies have examined the relationship between hyponatremia and antidepressants. ${ }^{18-23}$ However, most studies are small and observational and only few have had the power to examine whether specific antidepressants carry a higher or lower risk of hyponatremia. The number of case reports and small observational studies with hyponatremia concerning selective serotonin reuptake inhibitors (SSRI) is substantially higher than the number of case reports and observational studies with other antidepressants, but it is not clear whether this is due to a true difference in incidence of hyponatremia. ${ }^{24}$ A recent review concluded that current evidence suggests a relatively higher risk of hyponatremia with SSRIs and venlafaxine compared to tricyclic antidepressants (TCA) and mirtazapine, but for several antidepressants, data were insufficient to determine the risk of hyponatremia. ${ }^{24}$

The aim of this study was to examine the association between specific classes of antidepressant medication and hyponatremia and between specific drugs within these classes and hyponatremia. We examined the most commonly used drugs among TCA, SSRI, serotonin-norepinephrine reuptake inhibitors (SNRIs) and noradrenergic and specific serotonergic antidepressants (NaSSAs).

\section{METHODS}

By the time of birth or immigration, every Danish citizen gets a permanent personal identification number through the Danish Civil Registration System. This makes it possible to link information from multiple registers at the individual level. The current study is a register-based population study where we linked exposure to antidepressant therapy with occurrence of hyponatremia. We used information from the Danish Civil Registration System, the Danish National Patient Register, the Danish register of Medicinal Products Statistics and laboratory data. Since 1968, the Danish Civil Registration has been complete regarding information about dates of birth and death for all Danish citizens. ${ }^{25}$ The Danish National Patient Register contains records of all hospitalisations since $1977 .^{26}$ The Danish Register of Medicinal Products Statistics consists of all prescription claims from Danish pharmacies since 1995, including medicine strength, quantity and date. ${ }^{27}$ The prescriptions are classified according to the Anatomical Therapeutic Chemical system (ATC).

\section{Laboratory data}

Information concerning p-sodium was obtained from the LABKA database containing results of all blood samples from hospitalised patients and outpatients submitted for analyses to hospital laboratories in the Northern and Central Denmark. From the database, information on the date and result of the analysis was retrieved.

\section{Population}

All individuals born before 1998 and living in The North Denmark Region in the period from 1 January 1998 to 31 December 2012 were included. Individuals born after 1 January 1998 were excluded.

We continuously included individuals who immigrated to The North Denmark Region in the study period. Individuals were followed until end of study, emigration, death or an event of hyponatremia, whichever occurred first.

\section{Outcome assessment}

Primary outcome was an event of hyponatremia defined as plasma sodium (p-sodium) below $135 \mathrm{mmol} / \mathrm{L}$. Secondary outcome was severe hyponatremia defined as p-sodium below $130 \mathrm{mmol} / \mathrm{L}$. Since the prescription register does not include medicine administered during hospital stay, hyponatremia during hospitalisation was excluded; however, the first blood test after admission was included if it was drawn on the day of admission.

\section{Antidepressant therapy}

We decided to include only the most commonly used antidepressants in Denmark. Information regarding antidepressant therapy was retrieved from the Danish National Prescription Register and included the following drugs (ATC codes in brackets): Amitriptyline (N06AA09), Clomipramine (N06AA04) and Nortriptyline (N06AA10), Citalopram (N06AB04), Escitalopram (N06AB10), Fluoxetine (N06AB03), Paroxetine (N06AB05) and Sertraline (N06AB06), Duloxetine (N06AX21), Venlafaxine (N06AX16) and Mirtazapine (N06AX11), Mianserin (N06AX03). Treatment was defined in a timedependent manner; thus, we only considered individuals as exposed if they were covered by a prescription claim. Every prescription in the prescription register contains data on tablet strength and number of tablets. To determine exposure status, we defined minimum, maximum and typical daily dosage for each drug and tablet strength. On the basis of this information, we calculated length of treatment by creating an algorithm that used up to five consecutive prescriptions. These calculations were exclusively based on previous prescription claims. When it was a patient's first prescription claim, the typical daily dosage was used and the amount of claimed medications was divided by the daily dosage to determine length of treatment. When a patient who was covered by a previous prescription claimed a new one, we calculated a new daily dosage by dividing the amount of claimed medications in the previous period by the time between prescription claims. If the calculated new daily dosage was higher than the stated maximum dosage, this dosage was used instead. It was presumed that the remaining tablets were kept and 
consumed immediately after the last prescription coverage ended.

\section{Comorbidities and concurrent pharmacotherapy}

The following comorbidities were identified as possible confounders of the association (The International Statistical Classification of Diseases and Related Health Problems 10th Revision, (ICD10) codes in brackets): chronic lung (J40-47, J60-J70, J80, J82-J84, J95-J99), liver (K70-K77), kidney (N18, N19), and intracranial (I60-I69) diseases, heart failure (I50) and cancer (C). These comorbidities were accessed from the Danish National Patient Register. All comorbidities were identified from 1993 and throughout the study period. Furthermore, we identified treatment with antiepileptic drugs or diuretics since these treatments could potentially confound the association. As for the antidepressant drugs, we defined treatment in a time-dependent manner, and treatment length was calculated in the same way.

\section{Statistics}

The incidence rate ratio (IRR) of hyponatremia associated with exposure to antidepressant therapy was analysed in a time-dependent multivariable Poisson regression model. Before the analyses, we divided observational time into 1 year timebands. Age was updated at the beginning of each timeband and afterwards categorised into 10-year intervals. At the time of a new diagnosis or change in medication, the observational time was divided into a 'pre' and a 'post' period, ensuring a timely correct classification of comorbidities and exposure to pharmacotherapy. Hereafter, binary variables (current use or no use) were created for each antidepressant. Furthermore, we divided time after first prescription into five timebands: $0-14,15-30,31-90,91-180$ and $>180$ days.

We performed five different multivariable Poisson analyses: (1) estimating IRR for having p-sodium $<135$ with current use of antidepressants; (2) estimating IRR for having p-sodium <130; (3) estimating IRR for having p-sodium measured after initiating treatment with the different antidepressants; (4) estimating IRR for hyponatremia after initiating treatment (in each of the previously mentioned timebands) with one of the included antidepressants. Owing to few events in some of the strata in this analysis, we explored the association between hyponatremia and four classes of antidepressants (TCAs, SSRIs, SNRIs and NaSSAs); (5) estimating IRR for hyponatremia in the first p-sodium measurement after initiating treatment. For the unexposed individuals we used their first p-sodium measurement after inclusion. Lastly, we made a subgroup analysis, only including individuals who had p-sodium measured. To avoid conditioning on the future, this was done in a logistic regression analysis instead of a time-dependent regression. In this analysis, we estimated the ORs for hyponatremia with current use of antidepressants, as for the Poisson regression analyses an individual was only considered exposed if covered by a prescription claim. All analyses were adjusted for age (in 10-year intervals), sex, comorbidities and concurrent pharmacotherapy with diuretics, antiepileptic drugs and other antidepressants; furthermore, all Poisson regression analyses were adjusted for time.

All analyses were performed using SAS V. 9.2 (SAS Institute).

\section{RESULTS}

In total, 638352 individuals were included in the study, and they were on average followed for 10.98 years. Of these, $15.50 \%(\mathrm{n}=98976)$ initiated treatment with an antidepressant drug. An event of hyponatremia occurred in $72509(11.36 \%)$ individuals, and $8.90 \%(\mathrm{n}=6476)$ of these events happened during treatment with antidepressants. Population characteristics for individuals exposed to the different antidepressants during follow-up and for unexposed individuals are shown in table 1.

During follow-up, $60.28 \% \quad(n=403.109)$ of the study population had their p-sodium measured. $75 \%$ of individuals who started treatment with antidepressants had psodium measured at some point during follow up, but only $31 \%$ had p-sodium measured within the first 6 months after initiation of treatment. The percentage of individuals who had p-sodium measured within 6 months after initiating treatment varied between drugs; the data are available in online supplementary appendix A.

Figure 1 shows the association between use of specific antidepressants and an event of hyponatremia and the chance of having serum sodium measured while being in treatment with an antidepressant. Drugs associated with a high chance of having p-sodium measured were also strongly associated with the finding of hyponatremia. We therefore performed a logistic regression analysis in the subpopulation of individuals who had p-sodium measured. This subpopulation included 403109 individuals; $20.25 \%$ of these $(n=81618)$ were at some point in treatment with an antidepressant. Figure 2 shows the association between use of antidepressants and hyponatremia in this subpopulation. The pattern of associations between use of specific antidepressants and hyponatremia in the subpopulation is very similar to the results in the total population. Clomipramine (OR 4.15, CI 2.56 to 6.74) and nortriptyline (OR 2.44, CI 1.99 to 3.00) are strongly associated with hyponatremia, but there are few events leading to uncertainty in data and wide CIs. Mianserin (OR 1.25, CI 0.96 to 1.62), mirtazapine (OR 1.53, CI 1.39 to 1.69 ) and escitalopram (OR 1.12, CI 0.98 to 1.29$)$ have a weaker association with hyponatremia than with citalopram (OR 2.34, CI 2.20 to 2.48). In order to avoid possible bias due to some individuals having p-sodium measured several times and thereby possibly increasing the chance of catching an event of hyponatremia, we performed a Poisson analysis to estimate IRR of hyponatremia in the first measurement of p-sodium after having initiated treatment with an antidepressant. This 


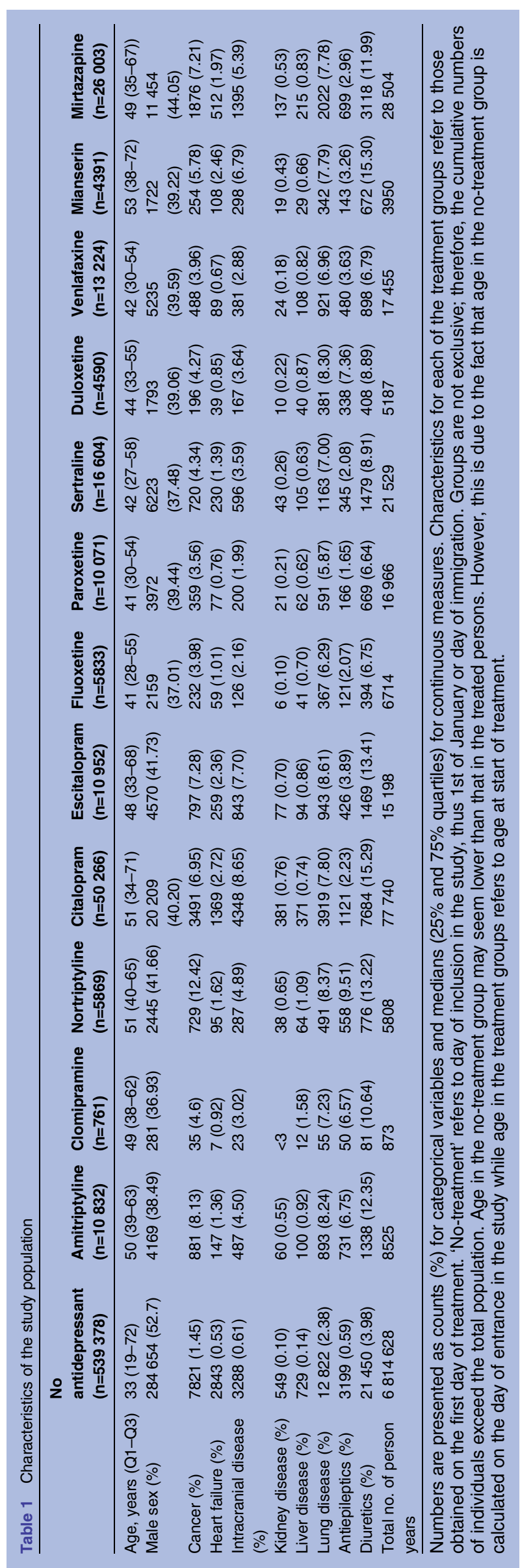

analysis showed a slightly different pattern with highest IRR for citalopram (7.80, CI 7.42 to 8.20); the results are shown in figure 3 . We also investigated the occurrence of hyponatremia relative to the time of initiating treatment with an antidepressant. For all antidepressants, the association between treatment and occurrence of hyponatremia was strongest in the first 2 weeks after initiation and thereafter gradually declined; the data are shown in figure 4.

Finally, we looked at the occurrence of severe hyponatremia, defined as p-sodium $<130 \mathrm{mmol} / \mathrm{L}$. The pattern of association was not different from mild hyponatremia, but due to fewer events the results are less clear with an overlap of CIs for most drugs; the data are available in online supplementary appendix B.

\section{DISCUSSION}

In this large register-based study including $\sim 10 \%$ of the total Danish population living in a geographically welldefined area (The North Denmark Region), we investigated the association between different antidepressants and incidence of hyponatremia. We observed that all the included antidepressants except mianserin were significantly associated with hyponatremia.

For all drugs, we found the highest IRR for hyponatremia during the first 14 days of treatment. This is consistent with previous studies ${ }^{28} 29$ and, with the assumed SIAD mechanism since the distribution and effect of $\mathrm{ADH}$, occurs within a few hours. This has been demonstrated in studies concerning thiazide where changes in plasma osmolality and p-sodium were observed within $6 \mathrm{~h}$ after a single dose of thiazide. ${ }^{30} 31$

\section{Definition of hyponatremia}

In this study, we defined hyponatremia as p-sodium $<135$. We have chosen this definition due to the fact that even mild hyponatremia during recent years has been shown to be of clinical importance.

Recently, an expert panel judged even mild hyponatremia to be of clinical importance due to association with adverse outcomes including mortality. ${ }^{32}$ A German study found mean p-sodium $131 \mathrm{mmol} / \mathrm{L}$ in psychiatric inpatients who were deemed to have symptomatic hyponatremia. Symptoms were primarily neuropsychiatric such as confusion, dizziness, gait impairments and falls. ${ }^{33}$ Hyponatremia due to any cause demonstrates an imbalance in the homeostatic system that normally controls p-sodium, and therefore even mild hyponatremia warrants cautiousness when considering medical treatment and medical advice concerning liquid and nutrition. One study even showed the level of p-sodium when patients were admitted to hospital to be associated with in-hospital mortality with a cut-off at p-sodium of $<138 \mathrm{mmol} / \mathrm{L}^{34}$

\section{Comparison of drug classes}

We have performed several analyses in order to minimise the bias that is inherent in an observational study. 


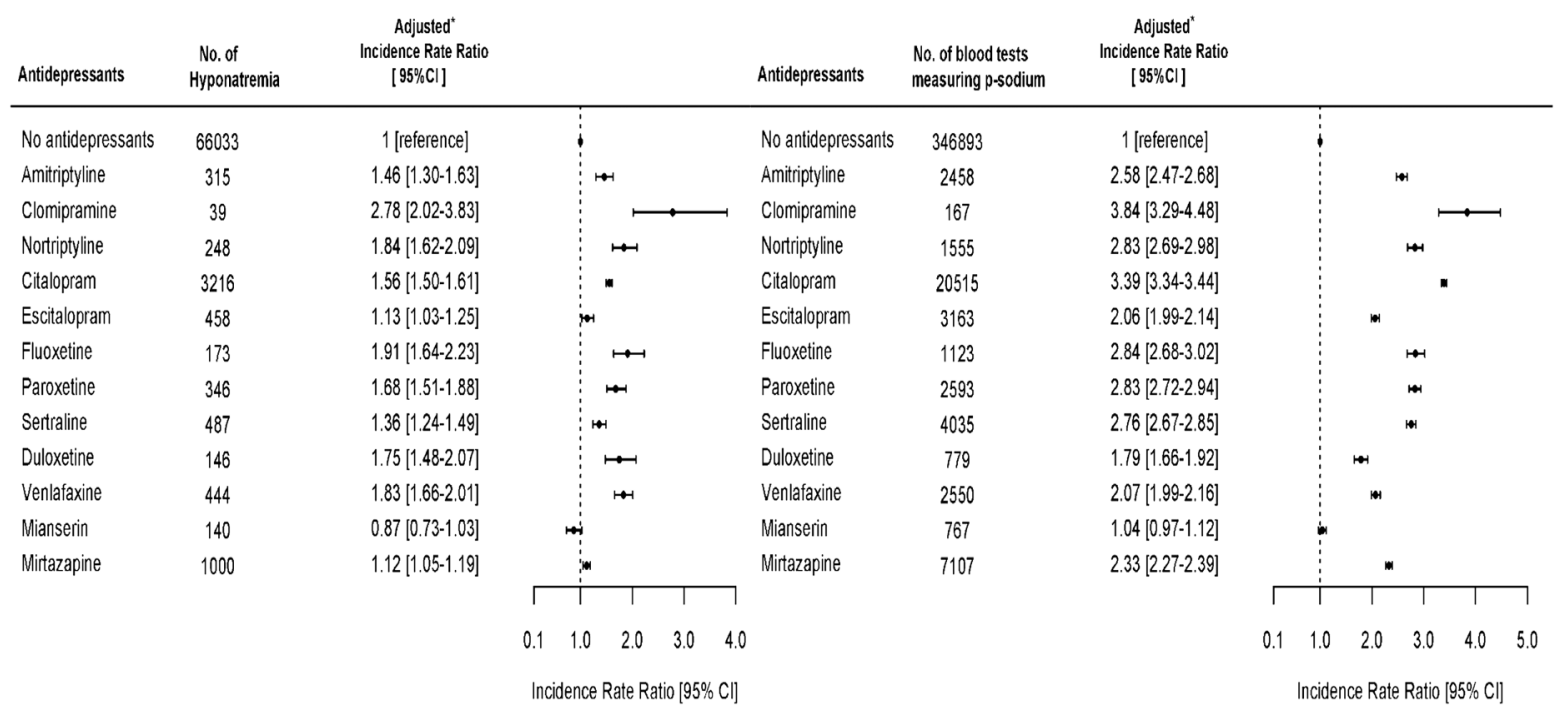

tAdjusted for age, time, sex, comoridities and concurrent pharmacotherapy with diuretics, antiepiliepic drugs and other antidepressants

Figure 1 Incidence rate ratios of having an episode of hyponatremia while being in treatment with an antidepressant and incidence rate ratios of having $\mathrm{p}$-sodium measured while being in treatment with an antidepressant.

Most of the analyses presented show similar results, but there are slight differences between the analyses presented. When comparing the specific drugs, we consider the analysis of association between drug treatment and hyponatremia in the first p-sodium measured to give the most valid and less biased results (figure 3). In this analysis, we have minimised the bias that may result from some individuals having p-sodium measured many times and some having p-sodium measured just once. Furthermore, on the basis of knowledge concerning pathophysiology of hyponatremia, we would expect hyponatremia due to an antidepressant to be present in the first p-sodium measured. TCAs and SSRIs in this analysis show IRRs for hyponatremia at the same level.
The initial reports of antidepressants associated with hyponatremia concerned TCA, but only a few later studies have compared the incidence of hyponatremia in TCAs with other antidepressants. In a large registerbased study, Coupland et al found a weaker association between hyponatremia and TCAs compared to SSRIs. However, Coupland's study included only persons above the age of 65 . We cannot therefore directly compare the results of Coupland's study with those of our study. ${ }^{23}$

The SNRIs including duloxetine and venlafaxine have lower IRRs. This is contradictory to another study that examined venlafaxine. ${ }^{21}$ However, this study had a very different population (patients in a psychiatric department) and only a few venlafaxine cases. To the best of

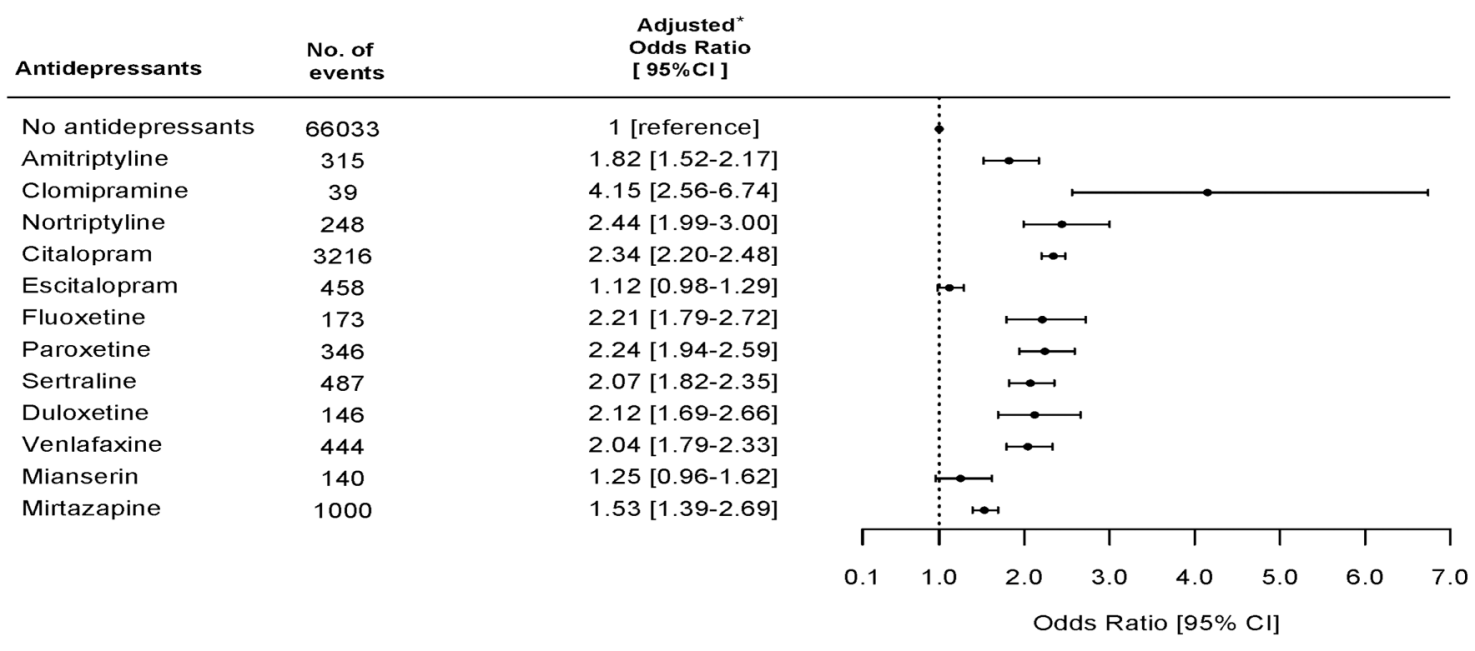

*Adjusted for age, sex, comorbidities and concurrent pharmacotherapy with diuretics, antiepileptic drugs and other antidepressants

Figure 2 ORs for hyponatremia for specific antidepressants in the subpopulation of individuals $(n=403109)$ who had $p$-sodium measured. 


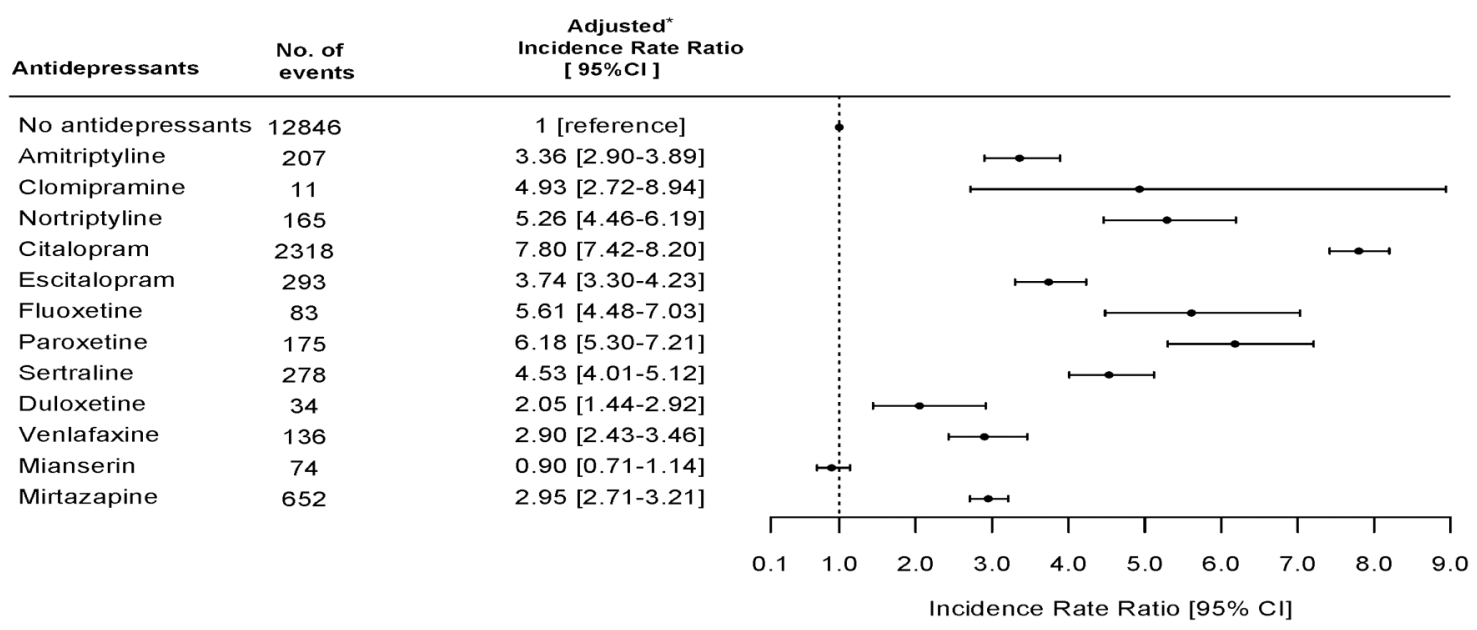

*Adjusted for age, time, sex, comorbidities and concurrent pharmacotherapy with diuretics, antiepileptic drugs and other antidepressants.

Figure 3 Incidence rate ratios for hyponatremia in the first measurement of $p$-sodium level after initiating treatment with a specific antidepressant.

our knowledge, duloxetine has previously been studied only in a case report including five cases. ${ }^{35}$

The NaSSAs include mianserin and mirtazapine. The IRR for mirtazapine was similar to that for duloxetine and venlafaxine. In contrast, mianserin was not associated with hyponatremia. On the basis of case reports and results from case series, it is generally assumed that mirtazapine has a lower risk of hyponatremia than SSRIs. ${ }^{20}{ }^{24}$ Our results support this assumption since the IRR for mirtazapine is significantly lower compared to SSRIs, but not compared to SNRIs. Mianserin and mirtazapine both block $\alpha 2$-adrenergic receptors, thereby increasing the level of norepinephrine. Interestingly, the $\alpha 2$-adrenergic system has been shown to be involved in polydipsia in patients with schizophrenia in a small pilot study where mianserin was used as treatment for polydipsia. ${ }^{36}$ The fact that mirtazapine also blocks postsynaptic serotonergic receptors might explain the difference between mianserin and mirtazapine.

\section{Mechanism of antidepressant-induced hyponatremia}

It is assumed that the mechanism of antidepressant hyponatremia is SIAD with or without elevated ADH. ${ }^{17}$ However, no studies have explored the influence of antidepressant-induced hyponatremia on thirst. One study has demonstrated that there is a downward setting of the osmotic threshold for thirst in patients with SIADH. ${ }^{37}$ Thiazide induced hyponatremia, which is also believed to be induced by an SIADH mechanism, has been associated with increased thirst. ${ }^{30}$ Combined with the assumption that the $\alpha 2$-adrenergic system is involved in polydipsia, this may explain why mianserin is not

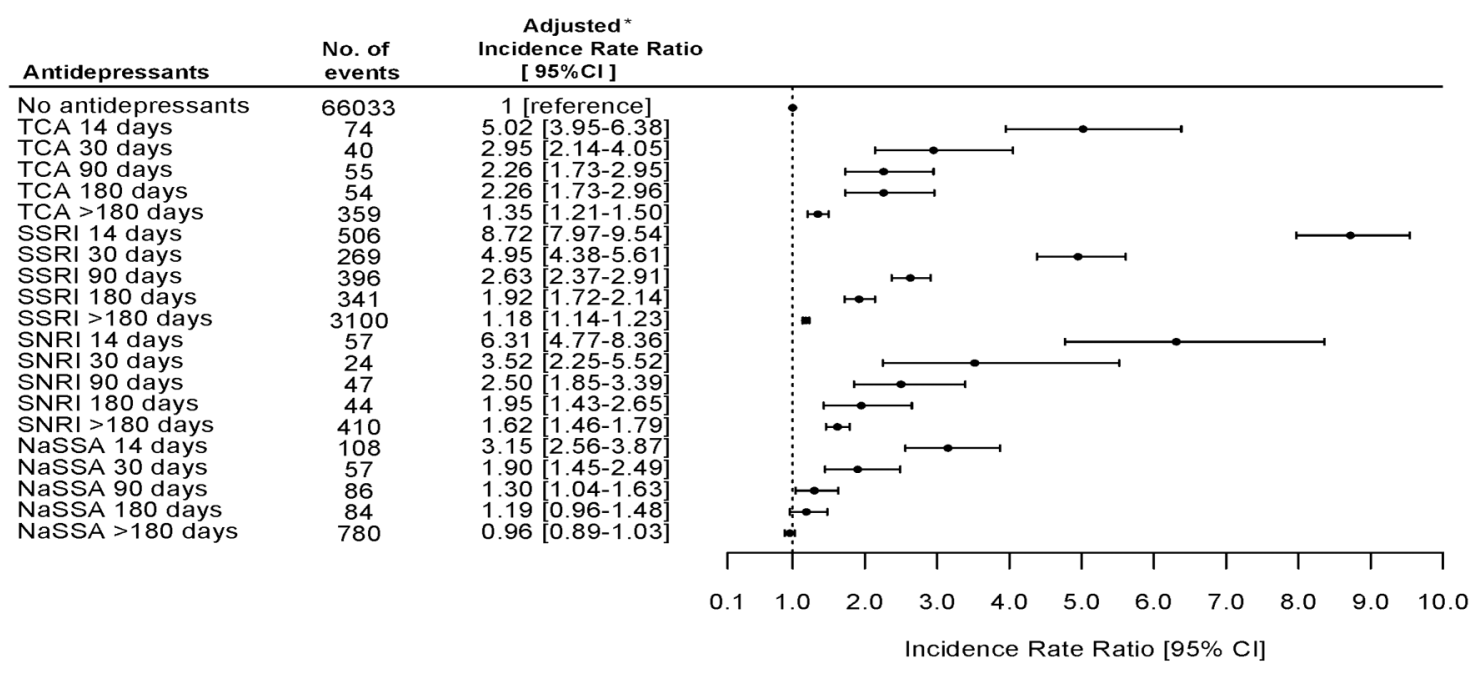

*Adjusted for age, time, sex, comorbidities and concurrent pharmacotherapy with diuretics, antiepileptic drugs and other antidepressants.

Figure 4 Incidence rate ratios for hyponatremia in timebands after initiating antidepressant therapy with drugs belonging to tricyclic antidepressants (TCA), selective serotonin reuptake inhibitors (SSRI), serotonin-norepinephrine reuptake inhibitors (SNRIs), and noradrenergic and specific serotonergic antidepressants (NaSSAs). 
associated with hyponatremia and mirtazapine to a lesser degree than other antidepressants.

\section{Clinical relevance and implications}

Incidence of hyponatremia increases with age, which is not surprising seen in the light of age-associated changes in water homeostasis. ${ }^{38} 39$ Even mild hyponatremia influences performance on cognitive as well as balance and mobility testing in elderly individuals. ${ }^{4} 5$ Chronic hyponatremia is associated with osteoporosis and high fracture risk. ${ }^{40}{ }^{41}$ Furthermore, mild hyponatremia is an indicator of a water balance disorder, which under different circumstances may develop into severe and life-threatening hyponatremia. This is underlined by the fact that patients, who for any reason have had hyponatremia once, are likely to have recurrent hyponatremia as a response to different diseases and stimuli, and lower baseline p-sodium is a risk factor for developing hyponatremia. ${ }^{42}{ }^{43}$ Even mild hyponatremia therefore warrants cautious evaluation and, if possible, should be abolished. The results from our study can inform the choice of antidepressants, especially when treating patients who are known to have a high risk of hyponatremia like, for instance, elderly people and persons who have previously had hyponatremia.

\section{Strengths and limitations}

The main strengths of this study are the large and welldefined population and high-quality registers, which made it possible to examine specific antidepressants. The limitations are mainly those that are inherent in an observational study, that is, residual confounding due to differences between patients and controls not accounted for because of lack of information on other variables that could possibly influence the risk of hyponatremia like, for instance, eating and drinking habits. Although we have adjusted for comorbidities and drugs known to be able to induce hyponatremia, we cannot fully exclude residual cofounding due to other reasons for hyponatremia. Not all persons who were treated with antidepressants had p-sodium measured and measurement may have been performed due to symptoms of hyponatremia. On the other hand, the symptoms of mild hyponatremia are often not recognised, and in elderly persons they may even be mistaken for symptoms of normal ageing (forgetfulness, dizziness, drowsiness). If the patients who had p-sodium measured represent a selection of more symptomatic patients, we would overestimate the rates of hyponatremia. However, an analysis including only those individuals in the population who had p-sodium measured showed similar results, which means that comparison between drugs should be safe. Thus, we consider the comparison of drugs valid, but we cannot be certain about the absolute level of IRRs for the specific drugs. The study population is Caucasian, and therefore generalisation of the results to different populations should be done with caution.
Comedication can increase or decrease the functional activity of hepatic cytochrome enzymes (CYP) and, as such, inhibit or induce the metabolic pathway of antidepressants. ${ }^{44} 45$ Since the CYP activity determines the plasma concentration of antidepressants and/or their metabolites that will be achieved as a function of the dose administered, drug-drug interactions may change the risk of dosedependent adverse drug events. In this study, potential drug-drug interactions were not taken into account.

\section{Conclusion}

There is an increased risk of hyponatremia for all included antidepressants, except for mianserin. The strongest association between hyponatremia and antidepressants is found in SSRIs. The association with SNRIs is slightly lower and the weakest association is with NaSSAs. The risk of hyponatremia is highest within the first 2 weeks of treatment. Considering the possible serious adverse effects of chronic hyponatremia, we recommend routine measurement of p-sodium shortly after initiating treatment with any antidepressant.

Contributors KBL-M, EAH, CT-P and GG designed the research. CT-P and GG were involved in the acquisition of data. KBL-M performed the research. All authors analysed the data. EAH, AHH and KBL-M wrote the manuscript. All authors contributed to a critical revision of the manuscript.

Funding This research was supported by a grant from the public Regional Research Foundation, Region Zealand.

Competing interests CT-P declares grants and personal fees from Cardiome, Merck, Sanofi and Daiichi, as well as grants from BMS, all outside the submitted work. GG is supported by an unrestricted clinical research scholarship from The Novo Nordisk Foundation. All other authors declare that they have no relationships with companies that might have an interest in the submitted work in the previous 3 years and none of the authors have non-financial interests that may be relevant to the submitted work.

Ethics approval This study was approved by the Danish Data Protection Agency (J.nr. 2007-58-015/l. Suite nr: Region H 00916 GEH-2010-001). In Denmark, no ethics approval is required for retrospective register-based studies.

Provenance and peer review Not commissioned; externally peer reviewed.

Data sharing statement Data stem from Danish National registers and cannot be shared.

Open Access This is an Open Access article distributed in accordance with the Creative Commons Attribution Non Commercial (CC BY-NC 4.0) license, which permits others to distribute, remix, adapt, build upon this work noncommercially, and license their derivative works on different terms, provided the original work is properly cited and the use is non-commercial. See: http:// creativecommons.org/licenses/by-nc/4.0/

\section{REFERENCES}

1. Pharmaceutical consumption 2013 [cited 2015 04.08.2015]. http:// www.oecd-ilibrary.org/sites/health_glance-2013-en/04/10/index.html? itemld=/content/chapter/health_glance-2013-41-en

2. Holland J, Bhogle M. Sertraline and mirtazapine as geriatric antidepressants. Psychiatr Danub 2013;25(Suppl 2): S286-90.

3. Gartlehner G, Thieda P, Hansen RA, et al. Comparative risk for harms of second-generation antidepressants: a systematic review and meta-analysis. Drug Saf 2008;31:851-65.

4. Gunathilake R, Oldmeadow C, McEvoy M, et al. Mild hyponatremia is associated with impaired cognition and falls in community-dwelling older persons. J Am Geriatr Soc 2013;61:1838-9.

5. Renneboog B, Musch W, Vandemergel X, et al. Mild chronic hyponatremia is associated with falls, unsteadiness, and attention deficits. Am J Med 2006;119:71.e1-8. 
6. Hannon MJ, Thompson CJ. The syndrome of inappropriate antidiuretic hormone: prevalence, causes and consequences. Eur J Endocrinol 2010;162:S5-12.

7. Hoorn EJ, Liamis G, Zietse R, et al. Hyponatremia and bone: an emerging relationship. Nat Rev Endocrinol 2012;8:33-9.

8. Terzian C, Frye EB, Piotrowski ZH. Admission hyponatremia in the elderly: factors influencing prognosis. J Gen Intern Med 1994;9:89-91.

9. Hoorn EJ, Zietse R. Hyponatremia and mortality: moving beyond associations. Am J Kidney Dis 2013;62:139-49.

10. Jamal SA, Arampatzis S, Harrison SL, et al. Hyponatremia and fractures: findings from the MrOS study. J Bone Miner Res 2015;30:970-5.

11. Hoorn EJ, Rivadeneira F, van Meurs JBJ, et al. Mild hyponatremia as a risk factor for fractures: the Rotterdam study. J Bone Miner Res 2011;26:1822-8.

12. Holland-Bill L, Christiansen CF, Heide-Jørgensen U, et al. Hyponatremia and mortality risk: a Danish cohort study of 279508 acutely hospitalized patients. Eur J Endocrinol 2015;173:71-81.

13. Usala RL, Fernandez SJ, Mete M, et al. Hyponatremia is associated with increased osteoporosis and bone fractures in a large US health system population. J Clin Endocrinol Metab 2015;100:3021-31.

14. Sharman A, Low J. Vasopressin and its role in critical care. Contin Educ Anaesth Crit Care Pain 2008:8:134-7.

15. Anderson RJ, Chung HM, Kluge R, et al. Hyponatremia: a prospective analysis of its epidemiology and the pathogenetic role of vasopressin. Ann Intern Med 1985;102:164-8.

16. Hoorn EJ, Zietse R. Hyponatremia revisited: translating physiology to practice. Nephron Physiol 2008;108:p46-59.

17. Mannesse CK, Jansen PA, Van Marum RJ, et al. Characteristics, prevalence, risk factors, and underlying mechanism of hyponatremia in elderly patients treated with antidepressants: a cross-sectional study. Maturitas 2013;76:357-63.

18. Degner D, Grohmann R, Kropp S, et al. Severe adverse drug reactions of antidepressants: results of the German multicenter drug surveillance program AMSP. Pharmacopsychiatry 2004;37(Suppl 1): S39-45.

19. Fabian TJ, Amico JA, Kroboth PD, et al. Paroxetine-induced hyponatremia in the elderly due to the syndrome of inappropriate secretion of antidiuretic hormone (SIADH). J Geriatr Psychiatry Neurol 2003;16:160-4.

20. Jung YE, Jun TY, Kim KS, et al. Hyponatremia associated with selective serotonin reuptake inhibitors, mirtazapine, and venlafaxine in Korean patients with major depressive disorder. Int $\mathrm{J}$ Clin Pharmacol Ther 2011:49:437-43.

21. Kirby D, Harrigan S, Ames D. Hyponatraemia in elderly psychiatric patients treated with Selective Serotonin Reuptake Inhibitors and venlafaxine: a retrospective controlled study in an inpatient unit. Int $J$ Geriatr Psychiatry 2002;17:231-7.

22. Letmaier $M$, Painold $A$, Holl AK, et al. Hyponatraemia during psychopharmacological treatment: results of a drug surveillance programme. Int J Neuropsychopharmacol 2012;15:739-48.

23. Coupland C, Dhiman P, Morriss R, et al. Antidepressant use and risk of adverse outcomes in older people: population based cohort study. BMJ 2011;343:d4551.

24. De Picker L, Van Den Eede F, Dumont G, et al. Antidepressants and the risk of hyponatremia: a class-by-class review of literature. Psychosomatics 2014;55:536-47.
25. Schmidt M, Pedersen L, Sorensen HT. The Danish Civil Registration System as a tool in epidemiology. Eur J Epidemiol 2014;29:541-9.

26. Lynge E, Sandegaard JL, Rebolj M. The Danish National Patient Register. Scand J Public Health 2011;39:30-3.

27. Kildemoes HW, Sorensen HT, Hallas J. The Danish National Prescription Registry. Scand J Public Health 2011;39:38-41.

28. Movig KL, Leufkens HG, Lenderink AW, et al. Serotonergic antidepressants associated with an increased risk for hyponatraemia in the elderly. Eur J Clin Pharmacol 2002;58:143-8.

29. Spigset O, Hedenmalm K. Hyponatremia in relation to treatment with antidepressants: a survey of reports in the World Health Organization data base for spontaneous reporting of adverse drug reactions. Pharmacotherapy 1997;17:348-52.

30. Friedman $\mathrm{E}$, Shadel M, Halkin $\mathrm{H}$, et al. Thiazide-induced hyponatremia. Reproducibility by single dose rechallenge and an analysis of pathogenesis. Ann Intern Med 1989;110:24-30.

31. Clark BA, Shannon RP, Rosa RM, et al. Increased susceptibility to thiazide-induced hyponatremia in the elderly. J Am Soc Nephrol 1994:5:1106-11.

32. Verbalis JG, Goldsmith SR, Greenberg A, et al. Diagnosis, evaluation, and treatment of hyponatremia: expert panel recommendations. Am J Med 2013;126:S1-42.

33. Lange-Asschenfeldt C, Kojda G, Cordes J, et al. Epidemiology, symptoms, and treatment characteristics of hyponatremic psychiatric inpatients. J Clin Psychopharmacol 2013;33:799-805.

34. Wald R, Jaber BL, Price LL, et al. Impact of hospital-associated hyponatremia on selected outcomes. Arch Intern Med 2010;170:294-302.

35. Kruger S, Lindstaedt M. Duloxetine and hyponatremia: a report of 5 cases. J Clin Psychopharmacol 2007;27:101-4.

36. Hayashi T, Nishikawa T, Koga I, et al. Involvement of the alpha2-adrenergic system in polydipsia in schizophrenic patients: a pilot study. Psychopharmacology (Berl) 1997;130:382-6.

37. Smith D, Moore K, Tormey W, et al. Downward resetting of the osmotic threshold for thirst in patients with SIADH. Am J Physiol Endocrinol Metab 2004;287:E1019-23.

38. Cowen LE, Hodak SP, Verbalis JG. Age-associated abnormalities of water homeostasis. Endocrinol Metab Clin North Am 2013;42:349-70.

39. Mannesse CK, Vondeling AM, van Marum RJ, et al. Prevalence of hyponatremia on geriatric wards compared to other settings over four decades: a systematic review. Ageing Res Rev 2013;12:165-73.

40. Tamma R, Sun L, Cuscito $C$, et al. Regulation of bone remodeling by vasopressin explains the bone loss in hyponatremia. Proc Natl Acad Sci 2013;110:18644-9.

41. Hannon MJ, Verbalis JG. Sodium homeostasis and bone. Curr Opin Nephrol Hypertens 2014;23:370-6.

42. Jacob S, Spinler SA. Hyponatremia associated with selective serotonin-reuptake inhibitors in older adults. Ann Pharmacother 2006:40:1618-22

43. Gefen S, Joffe E, Mayan $\mathrm{H}$, et al. Recurrent hospitalizations with moderate to severe hyponatremia in older adults and its associated mortality. Eur J Intern Med 2014;25:624-8.

44. Ereshefsky L, Jhee S, Grothe D. Antidepressant drug-drug interaction profile update. Drugs R D 2005;6:323-36.

45. van der Weide J, Hinrichs JW. The influence of cytochrome P450 pharmacogenetics on disposition of common antidepressant and antipsychotic medications. Clin Biochem Rev 2006;27:17-25. 